\title{
Unilateral Nevoid Telangiectasia in a Healthy Man
}

\author{
Kirley Küçük Florence Bourlond Nicolas Votquenne Farida Benhadou \\ Department of Dermatology, Hôpital Erasme, Université libre de Bruxelles, Brussels, \\ Belgium
}

\author{
Keywords \\ Unilateral nevoid telangiectasia · Dermatomes · Hyperestrogenism · Liver disorder · Myxoma
}

\begin{abstract}
We report the case of a healthy 26 -year-old man presenting telangiectatic macules on the left thorax and arm since childhood. The main diagnostic hypothesis were unilateral nevoid telangiectasia (UNT), hereditary benign telangiectasia, atrial myxoma, segmental serpiginous angioma, circumscribed neviform angiokeratoma, and nevus vascularis mixtus. The diagnosis retained was UNT characterized by congenital or acquired telangiectasia distributed asymmetrically along the upper extremities, or the third or fourth cervical dermatomes. The congenital form is extremely rare, predominant in men, and persists in adulthood. The acquired form is most frequent, affects preferentially women, usually appears at puberty or during pregnancy and tends to disappear. Estrogen excess triggers the formation of telangiectasia. UNT is rarely associated with liver or thyroid disorder. Pulsed-dye lasers and normalization of estrogen are proposed as therapeutic options. We report a rare diagnosis of UNT in a young man with no other underlying condition. We would like to highlight that in the presence of unilateral telangiectasia, a complete clinical examination must be performed to rule out signs of hyperestrogenism in man, ocular or neurological abnormalities, a blood test to exclude pregnancy, hepatic and thyroid dysfunctions, and ultrasonography in case of suspicion of atrial myxoma.
\end{abstract}

\section{Introduction}

Telangiectasia is a common condition that may affect many healthy people but can also be a sign of serious underlying conditions. We would like to discuss, through the presentation of a clinical case, a rare diagnosis of telangiectasia that may require deeper clinical investigations. 


\section{Case Report}

We report the case of a 26-year-old man presenting multiple asymptomatic cutaneous lesions on the left thorax and the left arm since childhood. The lesions have been growing in number since adolescence. The patient occasionally consumes alcohol and 40 cigarettes per week and has no relevant personal or familial medical history. The clinical examination revealed the presence of numerous telangiectatic macules disappearing with pressure and located regarding the left thorax and arm (Fig. 1, 2). The rest of the examination was normal, with no mucosal lesions and no other systemic symptoms. We proposed the diagnosis of unilateral nevoid telangiectasia (UNT), and other differential diagnosis were the segmental form of serpiginous angioma, circumscribed neviform angiokeratoma, hereditary benign telangiectasia (HBT), nevus vascularis mixtus, and atrial myxoma.

\section{Discussion}

The diagnosis of UNT was proposed for our patient, but other rare diagnoses were investigated. Serpiginous angioma and HBT begin in childhood. Serpiginous angioma is rare and results from a capillary malformation. There is a female predominance. The multiple lesions are red punctate in "poivre de cayenne" and partially disappear with pressure. They are typically distributed in clusters and sheets on the extremities [buttocks and thighs] with a serpiginous livedoid pattern. Two forms of serpiginous angioma can be distinguished: nonsegmental or segmental. The lesions of the segmental form may distribute along a dermatome. This form is sporadic, and the lesions may regress in adulthood [1]. Extracutaneous manifestations [ocular and neurological] are reported [2]. HBT is characterized by diffuse, linear, or arborescent red punctiform lesions on photo-exposed areas [face and trunk], possibly surrounded by anemic halo, without mucosal or systemic involvement. Circumscribed nevi form angiokeratoma is a rare congenital malformation. The hyperkeratotic lesions are brownish-red, from 1 to $5 \mathrm{~mm}$ and arranged in a band-like pattern [1]. Nevus vascularis mixtus consists of intermingled anemic nevus and reticular telangiectasias. The distribution is in a "checkerboard pattern of mosaicism" in a single segment but can be more widespread and cover the whole body. This disease can be associated with vascular brain lesions, called the mixed vascular nevus syndrome $[1,3]$. The atrial myxoma, a benign intracardiac tumor, is associated with long and coarse telangiectasias of the superior vena cava area. A cardiac ultrasonography must be performed to diagnose the disease. The telangiectasia disappears a few months after the removal of the tumor [4]. Differential diagnosis is discussed in Table 1.

Our case report was compatible with a diagnosis of UNT. UNT is characterized by congenital or acquired multiple persistent asymptomatic superficial blanching telangiectasias along dermatomes or Blaschko's lines [5] and by asymmetric skin involvement [6], but symmetric conditions have been rarely described $[1,2]$. The most affected sites are the third and fourth cervical dermatomes of the trunk and upper extremities [6]. The congenital form is very rare, seen in the neonatal period or infancy, predominant in men, and tends to persist throughout life. The most frequent form is the acquired form, predominant in women that usually appears at puberty or during pregnancy and tends to disappear spontaneously [6]. The acquired form may be triggered by an elevated level of or sensitivity to estrogen, resulting in increased angiogenesis and formation of telangiectasia $[1,7,8]$.

We can distinguish 2 morphologic variants resulting from post-zygotic mosaicism: the UNT of the patchy type and the unilateral punctate telangiectasia (UPT). UNT is characterized

\section{Karger'}


Fig. 1. UNT involving the left thorax and arm. UNT, unilateral nevoid telangiectasia.

Fig. 2. Multiple patch of telangiectasia in dermatoscopy.
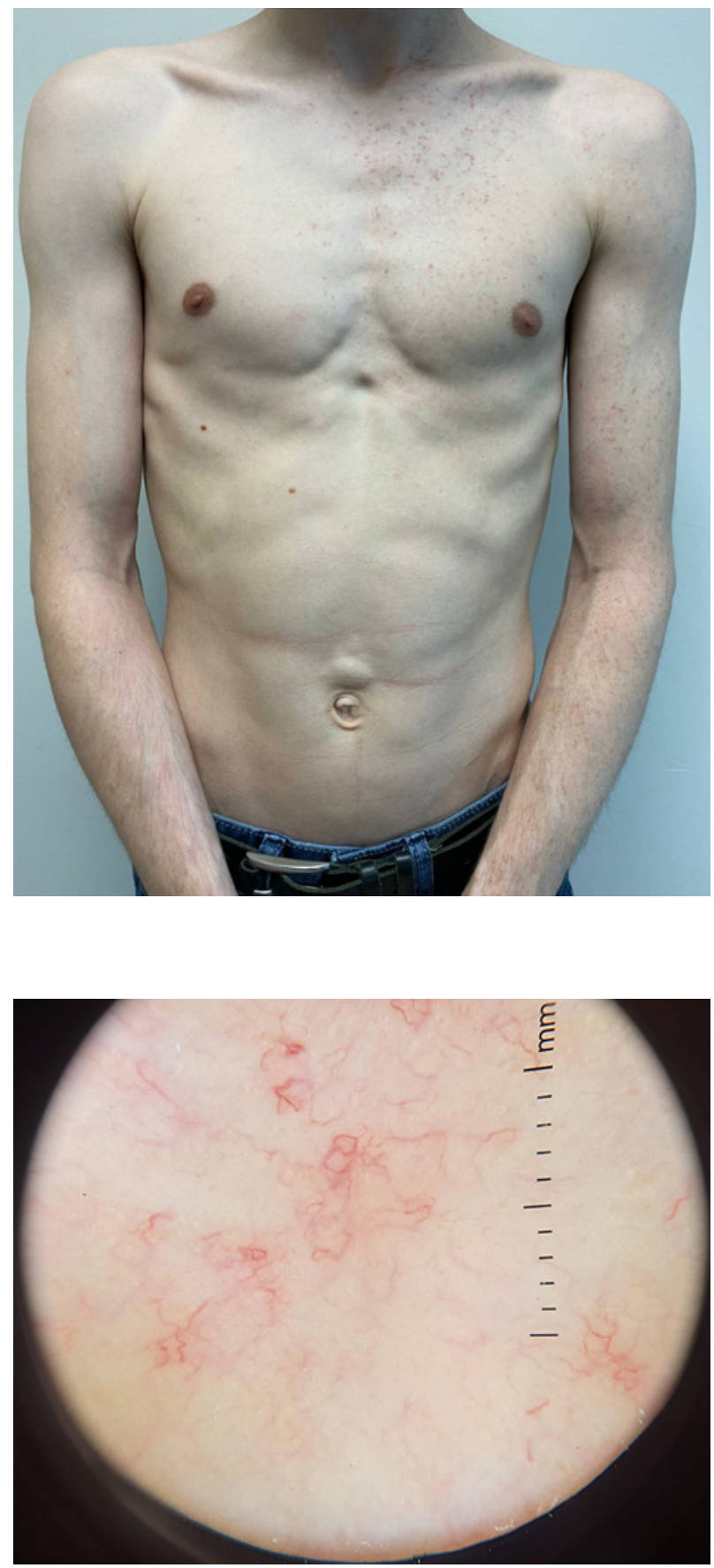

by unilateral punctiform telangiectasia surrounded by an anemic halo. The lesions may develop during childhood or adulthood and are frequently associated with liver diseases or hyperestrogenism. UPT is characterized by telangiectatic macules of various sizes which are located asymmetrically and follow Blaschko's lines. Hyperestrogenism can be present. Neurological disorders can be rarely associated with UPT. This association may be explained by a polygenic defect or a defect in the neurocutaneous developmental stages during embryologic life [1]. The distinction between UNT and UPT is complicated. Thereby, patients with UNT should be evaluated neurologically $[5,8]$. Our patient presents idiopathic unilateral punc- 
Case Reports

in Dermatology
Case Rep Dermatol 2021;13:330-335

DOI: 10.1159/000514941

(c) 2021

Küçük et al.: Unilateral Nevoid Telangiectasia

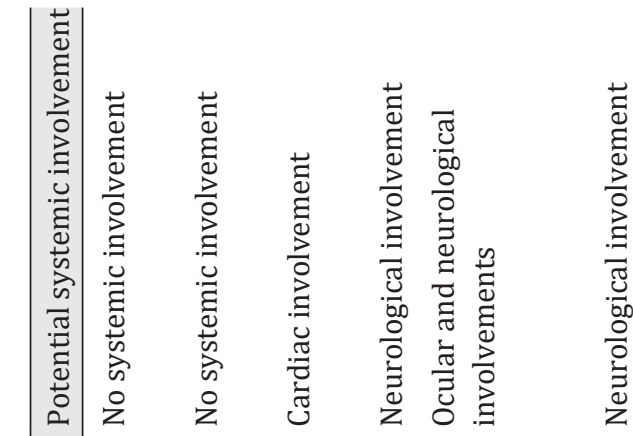

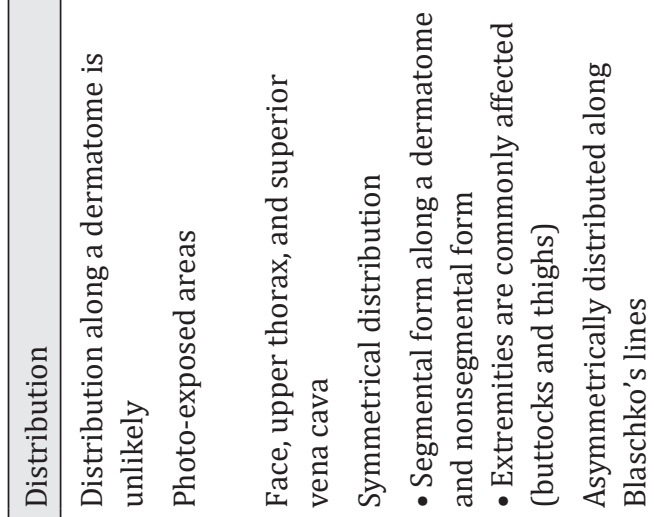

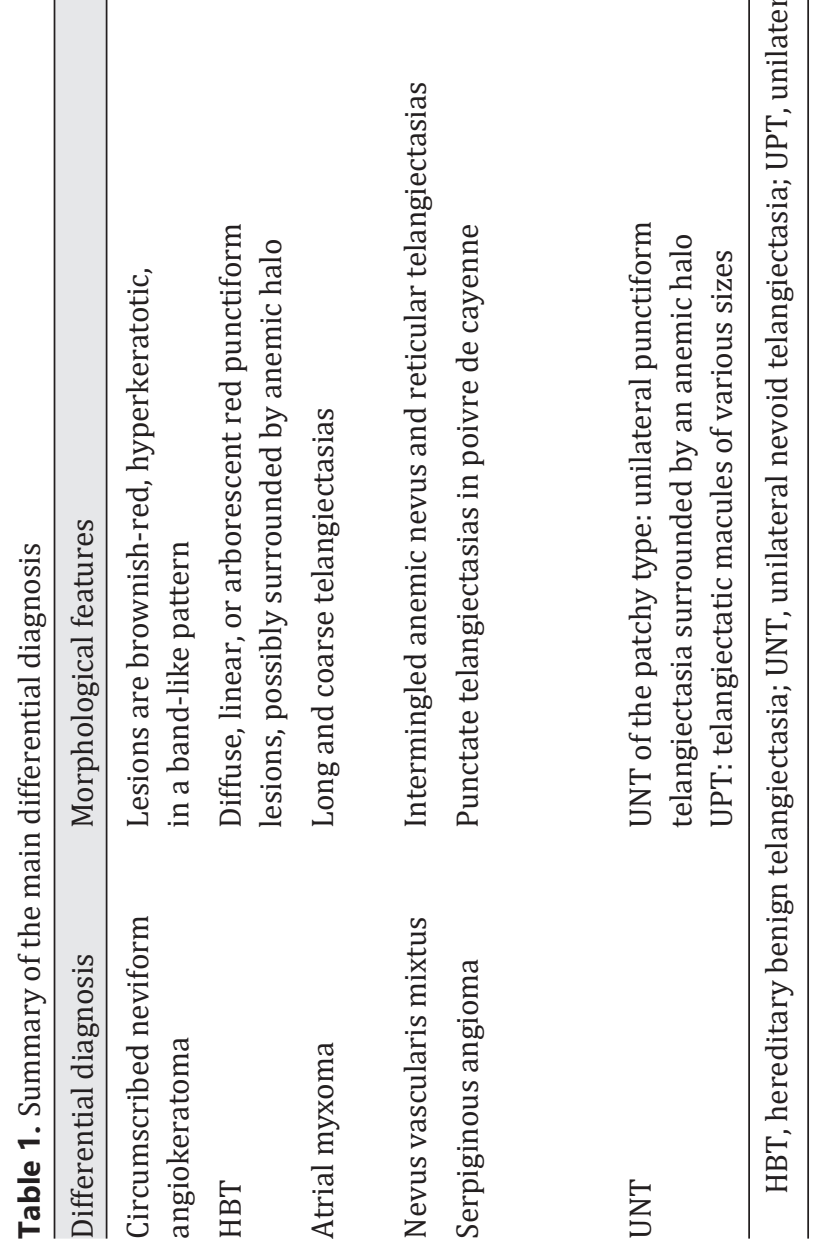


tiform telangiectasias since childhood without anemic halo. The lesions are distributed along a dermatome.

About 100 cases of UNT were associated with female sex [9], and only a few cases were reported among men [10]. Pulsed-dye lasers and normalization of the estrogen level are proposed as therapeutic options [6]. We report a rare diagnosis of UNT in a young healthy person with no other underlying condition. By the presentation of this case, we would like to highlight that while faced with unilateral telangiectasia, a complete clinical examination has to be performed to rule out signs of hyperestrogenism in men or ocular and neurological abnormalities, and a blood test has to be performed to exclude pregnancy and hepatic and thyroid dysfunctions. Ultrasonography will be performed in case of clinical suspicion of atrial myxoma.

\section{Statement of Ethics}

Written informed consent was obtained from the subject for publication [including images]. The case complied with the Declaration of Helsinki.

\section{Conflict of Interest Statement}

The authors have no conflicts of interest to declare.

\section{Funding Sources}

There was no funding, and the authors have no conflicts either actual or perceived.

\section{Author's Contributions}

Kirley Küçük: first author: contact with the patient, literature review, and manuscript writing. Florence Bourlond: contact with the patient, photography, and obtained written consent from the patient. Nicolas Votquenne: manuscript reviewing and literature review. Farida Benhadou: investigator: diagnosis and manuscript writing.

\section{References}

1 Happle R. Capillary malformations: a classification using specific names for specific skin disorders. J Eur Acad Dermatol Venereol. 2015;29(12):2295-305.

2 Erbagci Z, Erbagci I, Erkilic S, Bekir N. Angioma serpiginosum with retinal involvement in a male: a possible aetiological role of continuous cold exposure. J Eur Acad Dermatol Venereol. 2004;18:238-9.

3 Torchia D. Naevus vascularis mixtus and mixed vascular naevus syndrome. Clin Exp Dermatol. 2020;46: 34-41.

4 Hospital RP. Possible association of cutaneous telangiectasia. Am J Med. 1989 0ct;87(4):483-5.

5 Akman-Karakaş A, Kandemir H, Senol U, Unal A, Duman O, Ciftcioglu MA, et al. Authors reply: new clues on the path of understanding unilateral naevoid telangiectasia. J Eur Acad Dermatology Venereol. 2011;27: $258-9$.

6 Abbas O, Rubeiz N, Ghosn S. Extensive and progressive eruption in a young woman. Acquired unilateral naevoid telangiectasia (UNT) syndrome in an extensive distribution. Clin Exp Dermatol. 2010;35(3): e85-6. 
7 Woollons A, Darley CR. Unilateral naevoid telangiectasia syndrome in pregnancy. Clin Exp Dermatol. 1996; 21(6):459-60.

8 Coe JS, George SMC. Image gallery: unmasking of unilateral naevoid telangiectasia during puberty. Br J Dermatol. 2018;178(5):e331.

9 Blaise S, Kherat N, Seinturier C, Beani JC. Lésions télangiectasiques circonscrites au membre supérieur : penser au syndrome des télangiectasies nævoïdes unilatérales. J Mal Vasc. 2009;34(4):272-4.

10 Rodríguez-Martín M, Sáez M, Carnerero A, García-Bustínduy M, Guimerá F, Rodríguez García F, et al. Unilateral naevoid telangiectasia in a young man after chemotherapy: a simple coincidence or a new clinical association? J Eur Acad Dermatol Venereol. 2006;20:1001-2. 